\title{
ON A THEOREM OF S. BERNSTEIN-WIDDER*
}

\author{
BY \\ J. D. TAMARKIN
}

The present note is merely a comment to the preceding paper by $\mathrm{D} . \mathrm{V}$. Widder, and was suggested by the reading of its manuscript. It gives a simplified proof of the following important theorem discovered recently by S. Bernstein, and subsequently, but independently, by Widder, whose proof is based upon entirely different principles.

THEOREM. A necessary and sufficient condition that the function $f(x)$ should be completely monotonic in the interval $c<x<\infty$ is that

$$
f(x)=\int_{0}^{\infty} e^{-x t} d \alpha(t),
$$

where $\alpha(t)$ is a non-decreasing function of such a nature that the integral converges for $x>c$.

The sufficiency of the condition is obvious since

$$
f^{(n)}(x)=(-1)^{n} \int_{0}^{\infty} e^{-x t} t^{n} d \alpha(t), x>c \quad(n=0,1,2, \cdots) .
$$

Conversely let $f(x)$ be completely monotonic in the interval $c<x<\infty$. Let $a$ be an arbitrary constant greater than $c$ and set $c_{i}=f^{(i)}(a)$. It follows from the monotonic character of $f(x)$ that the quadratic form

$$
Q_{n}(x)=\sum_{i=0}^{n} \sum_{j=0}^{n} c_{i+j} x_{i} x_{j} \quad(n=0,1,2, \cdots)
$$

is non-negative. This fact is sufficient to ensure the existence of at least one non-decreasing function $\rho(t)$ such that $\dagger$

$$
c_{i}=\int_{-\infty}^{\infty} t^{i} d \rho(t) \quad(i=0,1,2, \cdots) .
$$

We now distinguish two cases:

CASE I. The function $\rho(t)$ is a step-function with a finite number of jumps.

CASE II. The function $\rho(t)$ is any other non-decreasing function.

* Presented to the Society, September 9, 1931; received by the editors June 13, 1931.

† See, for example, Marcel Riesz, Sur le probleme des moments, Arkiv för Matematik, Astronomi och Fysik, vol. 17, no. 16 (1923). 
CASE I. If $\rho(t)$ is a step-function with $p$ positive jumps at the points $-\lambda_{1},-\lambda_{2}, \cdots,-\lambda_{p}$ we have

$$
c_{m}=\sum_{k=1}^{p} \sigma_{k}\left(-\lambda_{k}\right)^{m}, \sigma_{k}>0 .
$$

From the Taylor development of $f(x)$ we obtain

$$
\begin{aligned}
f(x) & =\sum_{i=0}^{\infty} \frac{c_{i}(x-a)^{i}}{i !}=\sum_{i=0}^{\infty} \sum_{k=1}^{p} \sigma_{k}\left(-\lambda_{k}\right)^{i}(x-a)^{i} / i ! \\
& =\sum_{k=1}^{p} \sigma_{k} e^{\lambda_{k} a} e^{-\lambda_{k} x} .
\end{aligned}
$$

We can now show that all the $\lambda_{k}$ are positive or zero. It is only a matter of notation to suppose that $\lambda_{1}<\lambda_{2}<\cdots<\lambda_{p}$. Suppose that $\lambda_{1}$ were negative. We should have

$$
\begin{aligned}
f^{\prime}(x) & =\sum_{k=1}^{p} \sigma_{k} e^{\lambda_{k} a}\left(-\lambda_{k}\right) e^{-\lambda_{k} x} \\
f^{\prime}(x) e^{\lambda_{1} x} & =-\lambda_{1} \sigma_{1} e^{\lambda_{1} a}+\sum_{k=2}^{p} \sigma_{k} e^{\lambda_{k} a}\left(-\lambda_{k}\right) e^{-x\left(\lambda_{k}-\lambda_{1}\right)} .
\end{aligned}
$$

From the latter equation it is clear that $f^{\prime}\left(x_{0}\right) e^{\lambda_{1} x}$ tends to a limit as $x$ becomes infinite, in fact to the positive limit $-\lambda_{1} \sigma_{1} e^{\lambda_{1} a}$. But since $f(x)$ is completely monotonic for $x>c$ we have $f^{\prime}(x) \leqq 0$ and

$$
\lim _{x=\infty} f^{\prime}(x) e^{\lambda_{1} x} \leqq 0 .
$$

The contradiction shows that $\lambda_{1}$ must be positive or zero.* But equation (1) may be written in the form

$$
f(x)=\int_{0}^{\infty} e^{-x t} d \alpha(t),
$$

where $\alpha(t)$ is a non-decreasing function. Hence the theorem is established in Case I.

CASE II. If $\rho(t)$ is not a step-function then the quadratic form is not only non-negative but is a positive definite form. For,

$$
Q_{n}(x)=\int_{-\infty}^{\infty}\left(\sum_{i=0}^{n} t^{i} x_{i}\right)^{2} d \rho(t) \quad(n=0,1,2, \cdots),
$$

\footnotetext{
* That it may be zero is seen by the example $f(x)=1+e^{-x}$, which is certainly completely monotonic for all $x$.
} 
and this is clearly positive unless $x_{0}=x_{1}=\cdots=x_{n}=0$. It follows that

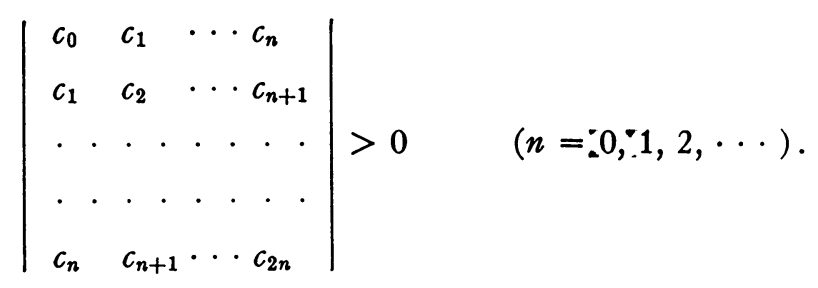

We may also show in this case that

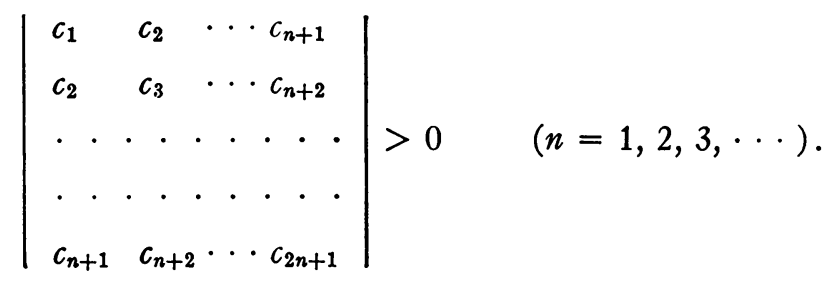

For, since $-f^{\prime}(x)$ is itself a completely monotonic function, the two cases applicable to $f(x)$ are also applicable to $-f^{\prime}(x)$. In the second of these cases we have (3) (which is merely (2) with all subscripts increased by unity). In the first of these cases we are led to a contradiction. For we should have

$$
\begin{gathered}
-f^{\prime}(x)=\sigma_{0}^{\prime}+\sum_{k=1}^{p} \sigma_{k}^{\prime} e^{\lambda k^{\prime} a} e^{-\lambda_{k^{\prime}} x}, \\
0<\lambda_{1}^{\prime}<\lambda_{2}^{\prime}<\cdots<\lambda_{p}^{\prime} ; \sigma_{0}^{\prime} \geqq 0, \sigma_{k}^{\prime}>0 \quad(k=1,2, \cdots, p) .
\end{gathered}
$$

Integrating equation (4) we should obtain

$$
f(x)=-\sigma_{0}^{\prime} x+\sum_{k=1}^{p} \sigma_{k}^{\prime} e^{\lambda_{k^{\prime}} a} e^{-\lambda_{k^{\prime}} x} / \lambda_{k}^{\prime}+C,
$$

where $C$ is a constant of integration. But $\sigma_{0}{ }^{\prime}$ must be zero, for otherwise

$$
\lim _{x=\infty} f(x)=-\infty \text {. }
$$

This is impossible since $f(x) \geqq 0$. But if $f(x)$ has the form (5) it is clear that the functions $f(x), f^{\prime}(x), f^{\prime \prime}(x), \cdots, f^{(p+1)}(x)$ are linearly dependent. Hence the Wronskian determinant of these functions must vanish identically. But this determinant reduces to (2) for $x=a, n=p+1$. We thus reach a contradiction. It follows that both (2) and (3) must hold in Case II. Hence we are in a position to apply a theorem of Hamburger* and obtain

* H. Hamburger, Bemerkungen zu einer Fragestellung des Herrn Polya, Mathematische Zeitschrift, vol. 7 (1920), p. 304. 


$$
f(x)=\int_{0}^{\infty} e^{-x t} d \alpha(t)
$$

where $\alpha(t)$ is a non-decreasing function. The theorem is thus established in all cases.

BROWN UNIVERSTTY, Providence, R. I. 